\title{
Maternal Near Miss : A Case Report
}

\author{
Serajun Noor ${ }^{1 *}$ \\ A M M Ehtashumul Hoque ${ }^{2}$ \\ Kawsar Alam ${ }^{3}$ \\ Nasir Uddin ${ }^{4}$
}

'Department of Obstetrics \& Gynecology

Chittagong Medical College

Chittagong, Bangladesh.

${ }^{2}$ Department of Nephrology

International Islamic Medical College Hospital

Chittagong, Bangladesh.

${ }^{3}$ Department of Aneasthesia

Chattagram Metropolitan (Pvt) Haspatal

Chittagong, Bangladesh.

${ }^{4}$ Department of Urology

Chittagong Medical College

Chittagong, Bangladesh.
*Correspondence to:

\section{Dr. Serajun Noor}

Associate Professor

Department of Obstetrics \& Gynecology

Chittagong Medical College

Chittagong, Bangladesh.

Mobile: +8801741652535

E-mail: noorserajun@yahoo.com

http://www.banglajol.info/index.php/CMOSHMCJ

\begin{abstract}
The term "Maternal Near Miss" (MNM) refer to women who have escaped death either by chance or due to good health care after experiencing severe life threatening complication during pregnancy, labour and within six weeks after termination of pregnancy. A "Near Miss" event in a $3^{\text {rd }}$ gravid at 42 weeks with intrauterine death having intractable Post Partum Haemorrhage (PPH) with severe maternal morbidity is reported here. Delay means death holds true for such emergency situation. The aim of the report is to stress the need of patients education, importance of emergency transportation and availability of multi-disciplinary tools and adequate blood transfusion at all level of health care system. Severe Acute Maternal Morbidity (SAMM) is a complement for maternal mortality and also to evaluate the quality of obstetric care in that particular institution.
\end{abstract}

Key word : Near miss; Post Partum Haemorrhage (PPH); Maternal mortality; Maternal morbidity; Maternal Death (MD); Pre Eclamptic Toxemia (PET).

\section{INTRODUCTION}

The object of Millennium Development Goals (MDGs) to improve maternal death by target $75 \%$ has not met with. Pregnant women health status is not reflected by mortality indication alone.

The concept of SAMM (Severe Acute Maternal Morbidity) is opt for the present health providing system. Near miss case review are one of the number of audit approach currently being used and evaluated with an interest in reducing higher rate of maternal mortality and to develop an understanding of the barrier as well as facilities surround the obstetric near miss cases.

The prevalence of near miss cases estimated to be 5.6 to $7.5 / 1000$ hospital based delivery and overall maternal near miss mortality ratio $9: 1^{1}$. Near miss case definition was based on validated specific criteria comprising of five diagnostic feature:- haemorrahge, hypertensive disorder of pregnancy, dystocia, sepsis and anemia $^{2}$.

Near miss approach for maternal health 2011 defined criteria for poor resource setting based on near miss management ICU, need for transfusion, near miss clinical criteria [PET,PPH] and organ dysfunction ${ }^{3}$. Review of near miss cases has the potential to highlight the deficiency and as well as the positive elements in the provision of obstetric service in any health system.

SAMM concept is superior over maternal death in drawing attention to surviving womens reproductive health and lives, and is equally applicable both in developed and developing countries ${ }^{4}$.

Maternal near miss incidence ratio, maternal near miss: mortality ratio (MNM:MD) mortality index are the near miss indices. High MNM:MD and low mortality index indicate better quality of health care ${ }^{5}$.

Maternal near miss cases are investigated over maternal death as:-

- Near miss are more common than maternal death. 
- near miss review is likely to yield useful information on same pathway that lead to severe morbidity and death.

- Investigating the care received may be less threatening to provide the cause of women survival.

- One can learn from women themselves since they can be interviewed about the care they received.

And patient herself is a source of data.

All the near miss should be interpreted as the free lesson and opportunity to improve the quality of service provision.

It is also clear that maternal death nearly are the tip of ice berg of maternal morbidity. High maternal mortality ratio may not allow detailed quantification of associated risk factor and determinants that are locally important .Underlying disease process of near miss and mortality were almost same so evaluation of circumstances surrounding near miss cases could act as proxy for maternal death ${ }^{6}$.

\section{CASE REPORT}

Mrs "A" 32 years old G3P2 with no living issue presented in labour at 42 weeks of pregnancy with intra uterine death on 18.6.13 at 8.am in Maa-O-Shishu Hospital. She had regular cycle, no ANC and was married for 8 years. She was normotensive, nondiabetic. She studied SSC level, belongs to lower middle socioeconomic stratum.

She delivered 2 hrs after her admission. Following delivery she had intractable PPH and with the diagnosis of atonic PPH management was done on estabilished management protocol.

Five units of blood was also transfused. But her condition was also deteriorated and she admitted in Chattagram Metropolitan Haspatal about 8 hrs after her delivery. On examination she was in shock (B.P./Pulse nonrecordable) severly anemic, respiration hurried, skin cold and calmy, pupil semidilated, slightly reacting to light and her clothings were soacked with blood.Her abdomen was soft with no distension,uterus soft and flabby, Vaginal bleeding continued in spite of condom in situ. Life support with dopamine, noradrenaline and Artificial ventilation was started.Fresh blood 4 unit and fresh frozen plasma 4 unit was also transfused.

Additional doses of uterotonic was given but bleeding still continued. After counselling patients condition and subsequent outcome, a decision of surgery was made under risk bond.CVP line started and under lifes upport laperotomy was done about $13 \mathrm{hrs}$ after her delivery. Abdomen was full of blood stained fluid, uterus was flabby, couvuleric, could not be contracted by any means, leading to uterine injury with compression suture. So peripartum hysterectomy was done but patient condition was unsatisfactory during and immediate after surgery. The patient was shifted to ICU under life support for the next $12 \mathrm{hrs}$ and her B.P was $60 / 40 \mathrm{~mm}$ of $\mathrm{Hg}$, pulse feeble, urine output was nil but bleeding stopped. About $20 \mathrm{hrs}$ after surgery the patient was gradually improving pulse 110 beats/min but feeble,B.P100/60mm of $\mathrm{Hg}$, Urine output gradually increased up to $800 \mathrm{ml}$ in next $24 \mathrm{hrs}$.

Extubation done and patient was shifted to HDU. On $3^{\text {rd }}$ POD her urine output decreased to $300 \mathrm{ml}$ in $24 \mathrm{hr}$ and diagnosed as acute renal failure (serum creatinine $5 \mathrm{mg} / \mathrm{dl}$, urea $60 \mathrm{mg} / \mathrm{dl}$, albumin $2 \mathrm{gm} / \mathrm{dl}$ ) and treated accordingly by nephrologist.
On $4^{\text {th }}$ POD she suddenly developed acute respiratory distress and a diagnosis of peripartum cardiomyopathy was made (ECHO-EF36\%, moderately severe left ventriculer dysfunction )and was treated by cardiologist. On $7^{\text {th }}$ POD she develop acute psychosis. On $10^{\text {th }}$ POD she had fever with loose motion (12 times/day,occult blood test $+v e$ ) and diagnosis of puerperal sepsis with multiorgan disease was made by medical board. Her wound was healthy but she had mild to moderate $\mathrm{p} / \mathrm{v}$ bleeding.

She developed jaundice on $12^{\text {th }}$ POD (billirubin $3 \mathrm{mg}$,ALT$1430 \mathrm{mIU} / \mathrm{dl}$,Albumin 2.6gm\%,PT-patient:control 21:13) and treated accordingly. She suffered from low grade fever(100$101 \mathrm{~F}$ ) and dysuria for about six weeks and treated with antibiotics for 2 months. She also had weakness of limbs from $2^{\text {nd }}$ weeks and was unable to walk without support ( obstetric palsy) and was improved with physiotherapy. She was discharged on $29^{\text {th }}$ POD with the advise to continue antibiotics and physiotherapy for 1 month.

She was under supervision of obstetrician and gynecologist and recovered completely within 3 months.

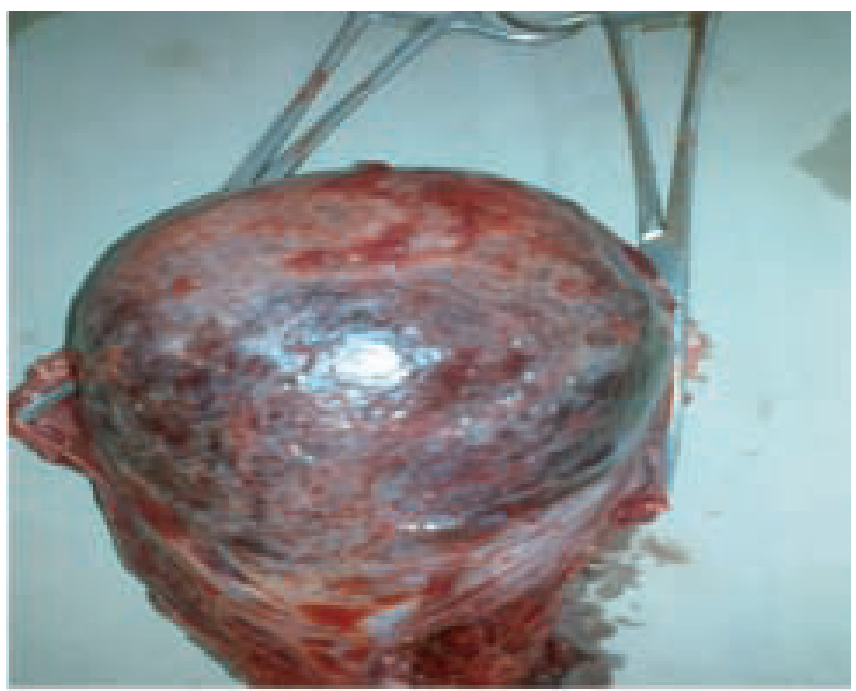

Figure 1 : Uterus during surgery

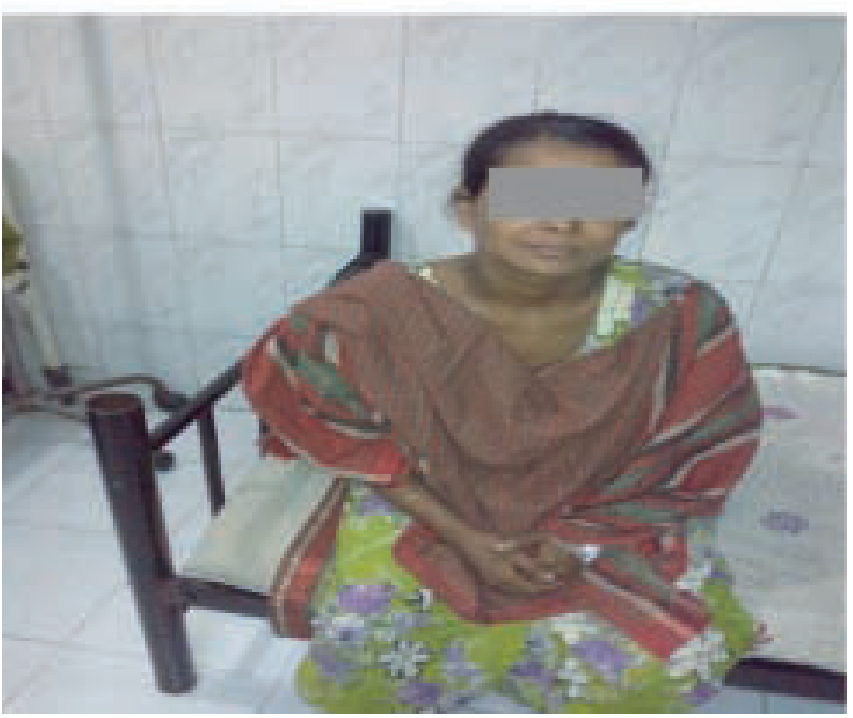

Figure 2 : Patient 28 days after surgery 


\section{DISCUSSION}

Obstetric emergency occur suddenly and unexpectedly endangering the life of mother and represent the quality of obstetric care. Present case is a classic example of maternal near miss. It illustrated importance of proper patient education, need for transportation, surgical and transfusion facility at community health centre, district and tertiary level to save maternal lives. Reducing maternal mortality is milleneum development goal. To reach the goal countries need an accurate picture of the cause and level of maternal death. The prospective of routinely using the accurate information for survelience of near miss cases, implementing preventive measure, timely intervention to avoid maternal death is promising.

PPH is still the most direct cause of maternal death $(31 \%$ in B.D) in the world ${ }^{7}$. Though most have some predisposing factor yet even without any factor unexpected deterioration in maternal condition that needs immediate attention, hospitalization, institutional active measure to control bleeding by medical, mechanical, nonsurgical and surgical intervention. Most of the maternal mortality occur within first $24 \mathrm{hr}$ following delivery .Effective treatment of $\mathrm{PPH}$ needs simultaneous multidisciplinary intervention.

Health care provider needs to begin immediate resuscitative effort, evaluate the cause of haemorrhage and take help of other care provider if required early referral to ICU.

Maternal near miss cases should be transferred to ICU with defined specific consensus of intensive care as lack of planning of intensive care may delay the implementation of necessary measure ${ }^{8}$.

In our patient delay in referral and surgical intervention resulted in serious morbidity leading to near miss situation. Avoiding delay in treatment could have a significant impact on survival.

Death with in $12 \mathrm{hrs}$ arrival in facilities indicate road block in seeking care or refferal ${ }^{8}$.

Data on cases of life threatening condition can be used to foster a culture of early identification of complication and better preparedness for acute morbidity even a remote rural area.

The knowledge about WHO near miss approach has to be spread to all the staff in health system-medical, paramedics, nonmedical and lower cadre to identify the problem and to help the patient in need. Apart from the maternal death maternal morbidity should be considered for disease analysis and to be reavealed to future generation so that the quality of care will be improved.
Signs of organ dysfunction that follows life threating condition are used to identify maternal near misses so that the classification of underlying causes is used for maternal death and near miss ${ }^{9}$. This consistency and a sets near miss indicators helps in assessment of quality care provided to pregnant women. It also helps to identify health system shortfall that countries need to address to reduce complication and fatal outcome of prenancy and child birth. All the district and maternity hospital should have facilities for surgery ,Banked blood component and ICU with sufficient trained stuff to handle life threatening complication to reduce severe maternal outcome. In SMH literature 3 delays are focused on obstetric care $^{9}$. Near miss review is a tool for maintainting the quality of maternal services in developing countries because persistently high level of maternal mortality has overshadowed the severe obstetric complication from which lesson could equally be learned. Crucial distinction between near miss upon arrival and after arrival is to be emphasized to have an addition information about prehospital barrier especially in home delivery and termination of pregnancy, even in the setting with available free maternal health care, transportation barrier, local health seeking behaviour while near miss after arrival usually provide information about within health facilities ${ }^{10}$. Resemblance of diagnostic pattern at facilities, maternal mortalities at regional level and reported similarities in substandard care, near miss has been suggested to act as surrogate analysis of maternal death ${ }^{11}$.

\section{CONCLUSION}

Severe acute maternal morbidity or near miss is a proindicator to improve quality of Obstetric care. Near miss are analysed to clarify the epidemiologic spectrum rapidly at hospital level and to prioritise the needs in maternal health care. Monitoring of near miss morbidity in conjunction with mortality surveillance could help to identify effective preventive measure for potentially life threatening morbidity. Severe maternal outcome can potentially be reduced by fostering the evidence based interventions for life threatening complications, improving referral systems and optimizing the use of critical care.

\section{DISCLOSURE}

All the authors declared no competing interest. 


\section{REFERENCES}

1. Maysoon Jabir,Imad Abdul Salam and Joao Paulo Souza:Maternal near miss and quality of maternal healthcare in Baghdad,Iraq BMJ. 2013:13:1-60

2. The WHO near miss approach for maternal health.GeneveWHO2011.

3. WHO,UNICEF,UNFPA and World Bank. Trends in maternal mortality:1990 to2008.Estimate developed by WHO.Geneva,Switzerland:WHO;2010

4. RonsmansC,Fillippi V:Reviewing severe maternalmorbidity: learning from survivors of life threatenening complications .Beyond the numbers:reviewing maternal death and complications to make Pregnancy safer WHO.Geneva:WHO.2004:103-123.

5. Hill K,ThomasK,AbouZahr C.WalkerN,Say L, Inou M,et al. Estimates of maternal mortality world wide between 1990 and2005 :an assessment of available data.2007;370:1311-1319.

6. Cecatti JG,Souza JP,Parpinelli MA,Sousa MH,Amaral E: Research on severe morbidities and near-misses in Brazil: What we have learned.Reprod Health Matters. 2007;15(30):125-133.

7. Tuncalp O,HindinM,Souza J,Chou D,SayL.The prevalence of maternal near : a systematic review.BJOG.2012;119(6):653-661.

8. Say L,Souza JP,PattitnsonRC.Maternal near miss towads astandard tool for monitoring quality of maternal health care.Best Pract Res Clin Obstet Gynaecol. 2009;23(3):287-296.

9. Fili ppiV,RonsmansC,GohouV.Maternity wards and maternity rooms? Incidence of near miss eventsin African hospitals.Acta Obstet Gynaecol Scand.2005;84(1):11-16.

10. ZeemanGG.Obstetric critical care:a blue print for improvedoutcomes.Crit care Med.2006;34(9Suppl):S208-S214 .

11. Pattinson RC,Hall M.Near misses:a usefull adjunct to maternal death enquires.Br Med Bull. 2003;67:231-243. 